\title{
Optimal Credit Card Customer Portfolio Construction Under Uncertain Situation
}

\author{
Xiaoxia Huang ${ }^{1,}$, , Xiaohong Zhao ${ }^{2, b}$ \\ ${ }^{1,2}$ Donlinks School of Economics and Management \\ University of Science and Technology Beijing, Beijing 100083, China \\ ahxiaoxia@manage.ustb.edu.cn, bxshiny920910@163.com
}

Keywords: Customer portfolio selection; uncertainty theory; credit loss

\begin{abstract}
Since the absence of portfolio model, banks may admit the credit card application without an effective evaluation standard in the extension of credit card business. This paper discusses a credit card customer portfolio selection problem in which the returns from customers are given by experts' estimations rather than historical data. The customer factors which directly affect the returns are regarded as uncertain variables. Based on customer segmentation, we proposed an optimal customer selection model with the objective of maximizing the expected return value within the constraints of capital limitation and risk diversification and the requirement on credit loss. To facilitate users to solve the model with practicable programming solvers, a crisp equivalent model is provided to show how credit card managers can make use of the model to select customers under the uncertainty theory.
\end{abstract}

\section{Introduction}

Portfolio selection involves optimal asset allocation and risk diversification. Modern financial portfolio is basically developed on Markowitz's mean-variance analysis [1], in which the expected return could be viewed as the investment return and the variance emphasized the risk reflects the degree of deviating from the expected return. Actually, this portfolio idea has been extended to other areas, like products [2], projects [3], even customers [4, 5, 6]. Customers should be regarded as risky assets, like stocks $[4,7]$. Why? Because the changes in consuming psychology will influence the size and frequency of one's expenditures and then make the firm's total cash flow fluctuate. Customer portfolio illustrates how a firm can maximize returns by attracting specific customers on the basis of how their consumption patterns act on the volatility of the whole cash flow. So the central principle of customer portfolio is balancing the return and risk of customers. Obviously, the return for customer portfolio is the profit that accrues to the firm. Like the analysis of financial risk, the customer risk can be measured by: customer beta [7] and variance of the cash flow [5]. Nevertheless, managers of Bank Credit should pay more attention to probability of default and credit loss resulting from default [6].

This paper attempts to build a well-behaved customer portfolio model to help banks better target new customers. As a media of convenient consumption and a source of revolving credit, credit card will bring profits accruing to banks from interchange, interest and fee income. As credit card loans are unsecured loans, we propose the proportion of bad customers of a group as the risk measure metric to help monitor the credit losses. Then we are able to examine the risk and return of each customer group and propose a customer portfolio to optimize the credit card customer structure under the constraints of capital limitation and risk diversification and credit loss control. Because of no historical data to describe a new customer's information, all of the incomes and the proportion of bad customers are uncertain. In 2007, Liu [8] proposed an uncertain theory to handle belief degree by uncertain measure facing the uncertain situation. Later on, Huang [9] systematically introduced the application of uncertainty theory in portfolio selection, since then a series of studies emerged: uncertain risk curve [10], uncertain mean-semivariance model [11], and mean-chance model [12]. In a word, this paper will deal with customer portfolio problem in the area of credit card business expansion by following Markowitz's mean-variance analysis and using the uncertain theory with the imprecise cash flows.

The rest of the paper is organized as follows. Section 2 will analyse the risk and uncertain incomes of credit card customer groups using the uncertain variables. Section 3 will propose a credit card 
customer portfolio model. Section 4 will give an equivalent of the model with the assumption that the incomes are all normal uncertain variables. Finally, we will give concluding remarks in section 5.

\section{Risk and revenue of credit card customer group}

The credit card business presents that capital is invested in credit card customer. To achieve the best benefits, banks need to find an effective way to target more profitable customer portfolio with lower risk, and decide how many customers should be acquired in each credit limit level. Our study focus on how credit card issuers identify an optimal customer structure in an uncertain environment.

Considering the complexity of the credit card business, this paper will provide several assumptions. Assumption 1, customers are the potential credit card customers but there is still no credit card statement. Assumption 2, all the credit card transactions will be carried on in a same billing period for each individual customer. Assumption 3, the customers have same credit limit are regarded as one group where each one has the similar transaction and repayment behaviors. Taken these factors into consideration, we can analyze credit card customer risk and return.

Risk. Generally, banks use a credit score to judge one's credibility degree. But here we propose the proportion of bad customers of a group as customer group risk measure metric which helps monitor a group's credit loss on the basis of customer segmentation. To illustrate, a bad customer is a guy who will have malicious overdraft without repayments. For each group $i$, the proportion is represented by $q_{i}$ and the credit limit is presented by $L_{i}$ When $i$ changes from 1 to $N$, the value of $q_{i}$ as well as $L_{i}$ is different from each other. It is noteworthy that $q_{i}$ is an uncertain variable with linear distribution.

Revenue. In the credit card operation, sources of incomes could be mainly summarized as the three categories. Interchange income: this income gained as a percentage of total transaction amounts from the merchants. Interest income: this part is the overdraft interest paid by credit card customers because of the delinquency in the payment during the previous period. Fee income: the total fees charged by banks from every link of using a credit card. Here we just take two main factors into consideration: cash advance and installment. Interchange income is highly correlated with one's total transaction while the interest income is determined by the interest-bearing balance in the previous period. However, fee income is affected by the cash advance amount as well as the total installments which are parts of the total amount of customers' transaction. Then we use $\alpha_{i}, \beta_{i}, \gamma_{i}$ respectively to denote the interchange, interest and fee incomes from each customer in ith customer group. To capture the relationships above, we propose the following mathematical expressions:

$$
\left\{\begin{array}{l}
\alpha_{i}=A_{i} \times a \\
\beta_{i}=B_{i} \times b \\
\gamma_{i}=C_{i} \times c+A_{i} \times \theta_{i} \times d
\end{array}\right.
$$

Where $A_{i}$ is the total transaction, $B_{i}$ is the interest-bearing balance in a previous period, $C_{i}$ is the withdrawal amount, $A_{i}, B_{i}, C_{i}$ are generated by one customer in ith group. $\theta_{i}$ is the proportion of installment of $A_{i}$. These four factors are independent of each other, and $A_{i}$ and $A_{j}$ independent of each other where $i \neq j$. And $a, b, c, d$ represents the constant average coefficient of those four factors.

Given the new customers' unknown behavior, we will make $A_{i}, B_{i}, C_{i}, \theta_{i}$ as uncertain variables to be estimated by experts under an uncertain situation. In reality, uncertain economic variables are likely to obey a normal uncertain distribution $N(\mu, \sigma)$. And we are able to deduce the parameters $\mu$ and $\sigma$ with the principle of $L S$ on the basis of the experts' estimation. The method of evaluating the value of uncertain variable has been introduced in Huang [16].

\section{Credit card customer portfolio selection model}

This paper aims at determining the size of each group by balancing the return and risk. We consider diversification program in $N$ customer groups, let $X_{i}$ be the rounded number of the ith customer group. As discussed in the revenue analysis, we notice that both interest and fee income are paid by 
customer himself, that means if there are $X_{i} q_{i}$ customers in the ith group default, the bank will lose these parts of the interest and fee income as well as the credit limit $L_{i}$. In reality, there also exist costs $L_{i} h$ which are related to the credit limit when maintaining a different customer. Then to maximize the expected net return under three certain constraints: risk diversification, budget and credit loss. We have the customer portfolio model as follow:

$$
\left\{\begin{array}{l}
\max E\left\{\sum_{i=1}^{N}\left[X_{i} \alpha_{i}+X_{i}\left(1-q_{i}\right)\left(\beta_{i}+\gamma_{i}\right)-X_{i} L_{i} h-q_{i} X_{i} L_{i}\right]\right\} \\
\text { subject } \text { to } \\
\quad V\left\{\sum_{i=1}^{N}\left[X_{i} \alpha_{i}+X_{i}\left(1-q_{i}\right)\left(\beta_{i}+\gamma_{i}\right)-X_{i} L_{i} h-q_{i} X_{i} L_{i}\right]\right\} \leq \kappa \\
\sum_{i=1}^{N} X_{i} L_{i} \leq L \\
E\left[\sum_{i=1}^{N} q_{i} X_{i} L_{i}\right] \leq M \\
X_{i} \geq 0, i=1,2, \cdots, N
\end{array}\right.
$$

Where $E$ denotes the expected value operator, $V$ is the variance operator of net returns, $\kappa$ the preset maximum tolerable volatility risk level, $L$ the total budget, and $M$ the preset maximum level of credit loss under control, $X_{i}$ the rounded number of the $i$ th customer group. And the variables $\alpha_{i}, \beta_{i}, \gamma_{i}$ can be represented by the four independent uncertain variables $A_{i}, B_{i}, C_{i}, \theta_{i}$ as the equation (1) shows.

\section{Equivalent of the model}

Before giving the equivalent model, we need to list the distribution of each uncertain variable. Here, we consider the quantity variables $A_{i}, B_{i}, C_{i}$ as normal uncertain variables and assume the ratio variables $\theta_{i}, q_{i}$ as linear uncertain variables. For ease of expression, we denote the normal uncertain variables by $A_{i} \sim N\left(\mu_{\left(A_{i}\right)}, \sigma_{\left(A_{i}\right)}\right), B_{i} \sim N\left(\mu_{\left(B_{i}\right)}, \sigma_{\left(B_{i}\right)}\right), C_{i} \sim N\left(\mu_{\left(C_{i}\right)}, \sigma_{\left(C_{i}\right)}\right)$, and linear uncertain variables by $\theta_{i} \sim L\left(e_{\left(\theta_{i}\right)}, f_{\left(\theta_{i}\right)}\right), q_{i} \sim L\left(e_{\left(q_{i}\right)}, f_{\left(q_{i}\right)}\right)$. Refer to Liu's definition [8] of the expected value and variance of uncertain variables, the customer portfolio model can be converted into the following one:

$$
\left\{\begin{array}{l}
\max \sum_{i=1}^{N} X_{i}\left[\mu_{\left(A_{i}\right)} a+\left(1-\frac{e_{\left(q_{i}\right)}+f_{\left(q_{i}\right)}}{2}\right)\left(\mu_{\left(B_{i}\right)} b+\mu_{\left(C_{i}\right)} c+\mu_{\left(A_{i}\right)} \frac{e_{\left(\theta_{i}\right)}+f_{\left(\theta_{i}\right)}}{2} d\right)-L_{i} h-\frac{e_{\left(q_{i}\right)}+f_{\left(q_{i}\right)}}{2} L_{i}\right] \\
\text { subject to } \\
\quad V\left\{\sum_{i=1}^{N} X_{i}\left[A_{i} a+\left(1-q_{i}\right)\left(B_{i} b+C_{i} c+A_{i} \theta_{i} d\right)-L_{i} h-q_{i} L_{i}\right]\right\} \leq \kappa \\
\sum_{i=1}^{N} X_{i} L_{i} \leq L \\
\sum_{i=1}^{n} X_{i} \frac{e_{\left(q_{i}\right)}+f_{\left(q_{i}\right)}}{2} L_{i} \leq M \\
X_{i} \geq 0, i=1,2, \cdots, N
\end{array}\right.
$$

Let $\eta_{i}=A_{i} a+\left(1-q_{i}\right)\left(B_{i} b+C_{i} c+A_{i} \theta_{i} d\right)-L_{i} h-q_{i} L_{i}$, and let $\Phi_{i}$ be continuous uncertainty distributions of the independent uncertain variables $\eta_{i}$, then $\eta_{i}$ has an inverse uncertainty distribution function: 


$$
\begin{aligned}
\phi_{i}^{-1}(\alpha)= & a\left(\mu_{\left(A_{i}\right)}+\frac{\sqrt{3} \sigma_{\left(A_{i}\right)}}{\pi} \ln \frac{\alpha}{1-\alpha}\right)+\left(1-\alpha \times e_{\left(q_{i}\right)}-(1-\alpha) f_{\left(q_{i}\right)}\right) \times\left(b\left(\mu_{\left(B_{i}\right)}+\frac{\sqrt{3} \sigma_{\left(B_{i}\right)}}{\pi} \ln \frac{\alpha}{1-\alpha}\right)\right. \\
& \left.+c\left(\mu_{\left(C_{i}\right)}+\frac{\sqrt{3} \sigma_{\left(C_{i}\right)}}{\pi} \ln \frac{\alpha}{1-\alpha}\right)+d\left(\mu_{\left(A_{i}\right)}+\frac{\sqrt{3} \sigma_{\left(A_{i}\right)}}{\pi} \ln \frac{\alpha}{1-\alpha}\right)\right)\left((1-\alpha) e_{\left(\theta_{i}\right)}+\alpha \times f_{\left(\theta_{i}\right)}\right)-L_{i} h \\
& -L_{i}\left(\alpha \times e_{\left(q_{i}\right)}+(1-\alpha) f_{\left(q_{i}\right)}\right)
\end{aligned}
$$

When the uncertain variables are represented by uncertainty distributions, the operational law of variance of uncertain portfolio return is given by Liu [13]. Finally, we are able to computing the variance value by employing the 9999 method in Huang [12].

\section{Conclusion}

This paper discusses a customer portfolio selection problem in the extension of credit card business. The returns from customers have to be predicted by experts' estimations because of no historical data. Uncertain variables are used to describe the factors which directly affect the returns. A mean-variance model for customer selection is proposed taking the capital limitation and risk diversification and credit loss into account under uncertainty situation. Moreover, we present the equivalent of the model when transaction amount and interest-bearing balance and withdrawal amount are normal uncertain variables while installment proportion and bad customer ratio are linear uncertain variables, so users are able to solve the model with available programming solvers.

\section{Acknowledgement}

This work was financially supported by National Natural Science Foundation of China (71171018) and Specialized Research Fund for the Doctoral Program of Higher Education (20130006110001).

\section{References}

[1] Markowitz.H, in: The journal of finance (1952).

[2] Cooper,R.G., Edgett,S.J., Kleinschmidt,E.J., in: Research-Technology Management (1997).

[3] Lawson, C.P., Longhurst, P.J., Ivey, P.C., in: Technovation (2006).

[4] Gupta, S., Lehmann, D.R., in: Journal of Interactive Marketing (2003).

[5] Tarasi, C.O., Bolton, R.N., Hutt, M.D., Walker, B.A., in: Journal of Marketing (2011).

[6] Singh, S., Murthi, B.P.S., Steffes, E., in: European Journal of Operational Research (2013).

[7] Dhar,R., Glazer,R., in: Harvard business review (2003).

[8] Liu, B. Uncertainty Theory, second ed. Springer-Verlag, Berlin, Chapter 5 (2007).

[9] Huang, X. Portfolio analysis: from probabilistic to credibilistic and uncertain approaches (Vol. 250). Springer Science \& Business Media. Springer-Verlag, Berlin, Chapter 4 (2010).

[10] Huang, X., in: Fuzzy Optimization and Decision Making (2011).

[11] Huang, X., in: Expert Systems with Applications (2012).

[12] Huang, X., Zhao, T., in: Insurance: Mathematics and Economics (2014).

[13] Liu, B. Uncertainty Theory: A Branch of Mathematics for Modeling Human Uncertain. Springer-Verlag, Berlin, Chapter 1 (2010). 